\title{
Effectiveness of two year balance training programme on prevention of fall induced injuries in at risk women aged 75-85 living in community: Ossébo randomised controlled trial
}

\author{
${ }^{1}$ Université Paris-Sud, \\ UMR-S1018, F-94807, Villejuif, \\ France \\ ¿Université Paris Descartes, \\ UMR-S 1153, F-75014, Paris, \\ France \\ ${ }^{3}$ Inserm, Centre de Recherche \\ Epidémiologie et Statistique \\ Sorbonne Paris Cité (CRESS), \\ U1153, F-94807, Villejuif, France \\ «UVSQ, UMR-S 1168, Université \\ Versailles St-Quentin-en- \\ Yvelines, France \\ 5Inserm, VIMA: Vieillissement et \\ Maladies Chroniques, U1168, \\ F-94807, Villejuif, France \\ ${ }^{6}$ AP-HP, Hôpital Sainte Périne, \\ Centre de Gérontologie, \\ F-75016, Paris, France \\ ${ }^{7}$ Conservatoire National des Arts \\ et Metiers (Cnam), Centre for \\ Research in Computer Science \\ and Telecommunications \\ (Cédric), EA4629, Paris, France \\ ${ }^{8}$ A1 AP-HP, Hôpital Ambroise Paré, \\ Unité de Recherche Clinique, \\ Département de Santé Publique, \\ Boulogne-Billancourt, France \\ Correspondence to: \\ P Dargent-Molina, Inserm \\ CRESS-équipe ORCHARD, \\ Hôpital Paul Brousse bâtiment \\ 15-16, 16 avenue Paul \\ Vaillant-Couturier, 94807 \\ Villejuif Cedex, France \\ patricia.dargent@inserm.fr \\ Additional material is published \\ online only. To view please visit \\ the journal online (http://dx.doi. \\ org/10.1136/bmj.h3830) \\ Cite this as: BMJ 2015;351:h3830 \\ doi: $10.1136 /$ bmj.h3830
}

Accepted: 18 June 2015

\author{
Fabienne El-Khoury, 1, 2,3 Bernard Cassou, 4, 5, 6 Aurélien Latouche,7 Philippe Aegerter,4, 5, 8 \\ Marie-Aline Charles, 2, 3 Patricia Dargent-Molina 2,3
}

\section{ABSTRACT \\ OBJECTIVE}

To assess the effectiveness of a two year exercise programme of progressive balance retraining in reducing injurious falls among women aged $75-85$ at increased risk of falls and injuries and living in the community.

\section{DESIGN}

Pragmatic multicentre, two arm, parallel group, randomised controlled trial.

\section{SETTING}

20 study sites in 16 medium to large cities throughout France.

\section{PARTICIPANTS}

706 women aged $75-85$, living in their own home, and with diminished balance and gait capacities, randomly allocated to the experimental intervention group (exercise programme, $\mathrm{n}=352$ ) or the control group (no intervention, $\mathrm{n}=354$ ).

\section{INTERVENTION}

Weekly supervised group sessions of progressive balance training offered in community based premises for two years, supplemented by individually prescribed home exercises.

\section{OUTCOME MEASURES}

A geriatrician blinded to group assignment classified falls into one of three categories (no consequence, moderate, severe) based on physical damage and medical care. The primary outcome was the rate of injurious falls (moderate and severe). The two groups were compared for rates of injurious falls with a "shared frailty" model. Other outcomes included the rates of all falls, physical functional capacities

\section{WHAT IS ALREADY KNOWN ON THIS TOPIC}

Exercise programmes emphasising balance training are effective in reducing falls in adults aged 65 and older living in the community, but randomised controlled trials have generally lacked the power to show an effect on injurious falls

Meta-analyses suggest that such programmes also prevent injuries caused by falls, including the most severe, but many interventions lasted only a few months, few targeted subgroups of older adults at highest risk of falls and injuries living at home, and the effectiveness of most interventions in real life conditions is unclear

\section{WHAT THIS STUDY ADDS}

The Ossébo trial contributes high quality evidence showing that it is feasible to put into place a large scale, long term (two year) exercise programme that is safe and effective in reducing injurious falls, even among at risk adults aged 75-85

The programme improved balance and gait capacities as well as quality of life related to physical function (balance and motor function test results), fear of falling (FES-I), physical activity level, and perceived health related quality of life (SF-36). Analysis was by intention to treat.

\section{RESULTS}

There were 305 injurious falls in the intervention group and 397 in the control group (hazard ratio $0.81,95 \%$ confidence interval 0.67 to 0.99 ). The difference in severe injuries (68 in intervention group $v 87$ in control group) was of the same order of magnitude $(0.83,0.60$ to 1.16). At two years, women in the intervention group performed significantly better on all physical tests and had significantly better perception of their overall physical function than women in the control group. Among women who started the intervention $(n=294)$, the median number of group sessions attended was 53 (interquartile range 16-71). Five injurious falls related to the intervention were recorded.

\section{CONCLUSION}

A two year progressive balance retraining programme combining weekly group and individual sessions was effective in reducing injurious falls and in improving measured and perceived physical function in women aged $75-85$ at risk of falling.

TRIAL REGISTRATION

ClinicalTrials.gov (NCT00545350).

\section{Introduction}

Injuries related to falls are common in older adults and are major contributors to functional decline and use of healthcare, ${ }^{12}$ including premature admission to nursing homes. ${ }^{3}$ Exercise programmes emphasising balance training are effective in reducing falls among older adults living in the community, ${ }^{45}$ but randomised controlled trials have generally lacked the power to show an effect on injurious falls.

We recently reviewed the current evidence on the effect of fall prevention exercise programmes among older adults living in the community on different outcomes of injurious falls, based on physical damage and medical care. ${ }^{6}$ Pooling data from 17 randomised controlled trials, we showed that programmes designed to improve balance and prevent falls can also prevent injurious falls, including those that cause fractures or other severe physical injuries, and can also reduce the rate of falls leading to medical care. But the methodological quality of several included studies could not be judged with any certainty, few studies used a predefined standardised classification of the consequences of falls, and some pooled analyses show significant heterogeneity between studies. Our review also highlighted 
the lack of comprehensive information about the programmes' impact on other important outcomes, especially psychological factors (such as fear of falling), health related quality of life, and potential adverse effects, although this knowledge is important in assessing the programmes overall benefit. ${ }^{7}$

Moreover, most interventions lasted less than one year, and few studies targeted the subgroups of older adults living at home, who are at highest risk of fall induced injuries, such as women aged over $75^{2}$-that is, those for whom the absolute benefit of the intervention should be greatest. ${ }^{8}$ Furthermore, the effectiveness of most of the interventions in real life conditions is unclear. Thus, designing and implementing interventions that are both effective against injurious falls and acceptable over the long term remains a challenge, especially for the oldest and more fragile subgroups. ${ }^{910}$

Ossébo is a large multicentre randomised controlled trial that sought to assess the effectiveness of a two year balance retraining programme in reducing injurious falls among women aged 75-85 living in the community with diminished balance or gait capacities. We also assessed the impact of the programme on physical as well as psychosocial outcomes (fear of falling, health related quality of life).

\section{Methods}

\section{Study design}

The Ossébo study is a multicentre, balanced, two arm, parallel group, randomised controlled trial. ${ }^{112}$ It applied a pragmatic approach ${ }^{1314}$ and aimed to assess the effectiveness of the intervention under real world conditions with existing community resources (instructors, settings).

\section{Eligibility and recruitment}

Women aged 75-85 living in the community were eligible if they had diminished balance or gait capacities, as assessed by the time they took to walk a 6 metre course (average of two measures) and the tandem walk test (ability to do four consecutive tandem steps). These two functional tests were used to select women for the trial because the EPIDOS (Epidémiologie de l'Ostéoporose) French cohort showed that they predict fall related fractures in women aged over 75 living in the community, ${ }^{15} 16$ and because they are simple and can be used easily by general practitioners and other health and physical activity practitioners. we planned to recruit women who were neither too fit nor too frail-that is, women at moderate risk of injurious falls who are most likely to benefit from the exercise programme we proposed (group based, weekly sessions, long duration). Women who took $\geq 7$ seconds to walk $6 \mathrm{~m}$ (median time in the EPIDOS cohort) or were unable to do four consecutive tandem steps were eligible. We excluded the frailest women-that is, those that took $>12.5$ seconds (95th centile in the EPIDOS cohort) to walk $6 \mathrm{~m}$ or were unable to stand for 10 seconds with their feet togetherbecause they were considered to be at high risk of falling and would probably require an individualised exercise programme (these women were referred to their general practitioner for further clinical evaluation). We also excluded women with medical conditions involving the neuromuscular, skeletal, or cardiovascular systems, which generally preclude exercising. These women were identified during baseline examination based on the following question: "are you currently being treated for heart disease, coronary disease, or joint pain that still bothers you in daily life despite the treatment." Other exclusions included women who were expected to move away within the next six months or would have difficulty attending exercise classes regularly, as well as women who were already taking exercise classes (to determine the true effect of the intervention).

The study took place in 20 sites in 16 medium to large cities throughout France. Recruitment was centrally organised (at Inserm CESP). Voter registration lists were used to identify all women aged 75-85 living in the community near the study sites. They received letters inviting them to a free balance and health examination. Women who returned the prepaid reply coupon were contacted by telephone to set up an appointment for the examination and were sent a questionnaire (on current medical treatments and health related quality of life, ${ }^{17}$ in particular) to be returned at the examination. The examinations took place in local hospital geriatric departments or senior health centres and were performed by examiners (usually nurses) specially recruited and trained for the study. They included the two tests used to determine trial eligibility (timed $6 \mathrm{~m}$ walk and tandem walk), other standardised functional tests of balance and motor function (series of static balance tests with feet in semi-tandem/tandem stand/side by side positions, get up and go test, one leg balance, five chair stands), ${ }^{1518-20}$ anthropometric (weight, height) and visual acuity measurements, and a structured face to face questionnaire on history of falls and fractures, fear of falling (FES-I), ${ }^{21}$ physical activities (average weekly frequency and amount of walking activities and sports and leisure activities over the past month), ${ }^{223}$ and sociodemographic characteristics.

\section{Randomisation}

Randomisation was centrally organised (at the ParisOuest Clinical Research Unit). The randomisation lists were computer generated, based on randomly permuted blocks of varying size (2, 4 or 6 , randomly sampled with equal probability). Randomisation was stratified for study centre and body weight $(<59 \mathrm{~kg} v$ $\geq 59 \mathrm{~kg}$ ), a major risk factor for low bone mineral density and fractures in older women, ${ }^{24}$ with a $1: 1$ ratio between the intervention and control groups. A biostatistician who had no other involvement in the trial generated the randomisation lists in advance, which were installed together with the examination data entry programme on laptops (one per centre, for study use only) before the opening of each centre. At the end of the baseline examination, the programme automatically determined the eligibility of each woman, based on her examination results; if she was eligible and 
agreed to participate, it randomly assigned her into the experimental intervention or the control group.

\section{Intervention}

The intervention was conceived and implemented in partnership with SIEL Bleu, a non-profit organisation specialised in delivering physical activity programmes for older adults (sielbleu.org). It involved free supervised sessions in small groups supplemented by individually prescribed home exercises. Group sessions of one hour were offered once a week for about two years in accessible community based premises (usually senior citizens clubs). Intervention instructors were all regular employees of SIEL Bleu, specifically trained for the study.

The general objectives of the Ossébo programme were to improve physical factors that affect balance and the risk of falling and injury from falls, to raise awareness of falling risks and ways of reducing them through behavioural changes, and to foster long term maintenance of regular physical activity for fall prevention through the integration of some exercises and healthy behaviours into participants' daily routine. The programme's technical content was based on a literature review (including detailed description of programmes requested from various authors, ${ }^{25-29}$ reference books on exercise for fall management, ${ }^{30}$ and SIEL Bleu's expertise and field experience. It was divided into eight terms of about 12 sessions, each with specific objectives and a standardised framework (combination and sequence of exercises) (appendix 1 provides a detailed description). The exercises were designed to improve postural stability (assessed by body sway), muscle extensibility and to a lesser degree joint flexibility (for example, hip flexor and calf stretches), balance (for example, knee bends, tandem stance, backward walking, sit to stand), reaction time (for example, play in group with a ball), coordination (for example, side leg swings, front leg swings), muscle strength critical for posture and balance (for example, hip abductor, knee extensor, ankle plantar-flexors), and internal sense of spatial orientation (senses of position and movement of limbs and trunk). Participants were also expected to perform exercises at home at least once a week to reinforce the group sessions and foster the integration of balance training and physical activity into the routines of daily living for a healthier lifestyle. The home exercises (about six) were selected from those practiced with the group and adapted by the instructor to each participant's physical abilities.

Instructors recorded attendance and reported frequency of home exercising at each group session and sent this information to the study coordination centre every week. They also reported any falls or other adverse events that occurred during group exercise sessions to the study clinical coordinator (BC), who then contacted the participant and her family physician. One person from SIEL Bleu (D Lutz, product developer) was responsible for supervising the instructors' work over the entire intervention period (such as through regular conference calls with instructors) to ensure the programme's satisfactory implementation in all centres.

\section{Control group}

Women randomised to the control group did not receive the Ossébo exercise programme. At the end of the baseline examination, all the women, included in the trial or not, were offered brochures about fall prevention (in particular those published by the French national institute of health prevention and education), which discussed the importance of physical activity, a balanced diet, and vitamin D supplementation and offered suggestions for assessing home hazards and managing drugs. Participants in both groups received newsletters twice a year reminding them about major risk factors for falls and prevention measures. At the end of the trial, participants in the control group were offered four free exercise sessions.

\section{Outcomes}

Falls were defined as "unexpected event[s] in which the participant comes to rest on the ground, floor, or other lower level." F Falls associated with unavoidable major events (such as stroke, traffic incident etc) were not considered in the analyses. The main outcome of the study was the rate of injurious falls (serious and moderate). Serious falls were those that caused fractures; head injuries requiring admission to hospital; joint dislocations; sprains accompanied by reduced physical function; other non-specified serious joint injuries; and lacerations requiring sutures. ${ }^{31}$ Injurious falls were classified as moderate if they resulted in bruising, sprains, cuts, abrasions, or reduction in physical function for at least three days or if the participant sought medical help. ${ }^{8}$

We also examined the rate of all falls, regardless of the consequences. Other secondary outcomes include physical functional capacity (balance and motor function tests results), fear of falling (FES-I score), ${ }^{21}$ physical activity level (casual walking, walking for exercise, and total leisure physical activities, all defined by weekly frequency and duration), ${ }^{22} 23$ and perceived health related quality of life (HRQoL; physical functioning, mental health, vitality, and general health components of the SF-36) ${ }^{17}$ measured at mid-term (after one year of intervention) and at the end of the intervention (after two years).

Monitoring the occurrence of falls during follow-up was centrally organised (at Inserm CESP) and was based on the calendar card method. ${ }^{7}$ Participants were asked to mark the exact date of any fall on pre-addressed, prepaid monthly calendar postcards, and to return the cards at the end of the corresponding month. Whenever a fall was reported, an investigator blinded to allocation group called the woman to conduct a standardised fall interview to confirm the fall and collect further information on its circumstances and consequences. If a fracture or admission to hospital was reported, a copy of the radiologist's report or medical record was requested to confirm the severity of the injuries. An investigator called every woman who did not 
return the card any month (or a person from her network of family or friends whose contact information was provided to us at inclusion).

Falls were then classified by an expert geriatrician (BC) blinded to group assignment into one of three categories: falls with no consequences, falls resulting in moderate injuries, and falls resulting in serious injuries. When needed, classification was reviewed with two other investigators, also blinded to group allocation (PDM and FEK), and the decision was reached by consensus $(n=25)$. We relied on the participant's narration of the fall during the fall survey, completed by specific standardised questions (such as: "was the fall due to a traffic incident," "were you overturned, shaken or struck before the fall," "did you lose consciousness just before falling," etc) to judge whether the fall was associated with a major intrinsic or extrinsic event. We excluded six injurious falls (two in the control group and four in the intervention group).

Participants were invited to the examination centre for one and two year follow-up examinations, conducted according to the same protocol as the baseline examination.

\section{Patient involvement}

No patients were involved in the design of the study (choice of inclusion criteria, local recruitment strategy, definition of outcomes, intervention design). These decisions were made by clinical geriatricians with special expertise in falls prevention and physical activity. No patients were involved in plans for recruitment or study conduct. At the end of the study, all participants were invited to a special informal and convivial gathering with the principal investigators (BC, PDM), the coordinator of the local examination centre, and the local SIEL Bleu instructor. The principal results of the trial will be disseminated to the study participants through the study newsletters.

\section{Change in the protocol}

We originally intended to recruit 1000 women in each group, which would have allowed us to detect a 30\% reduction in serious falls in the intervention group with a statistical power of $80 \%$ (assumptions: eight injurious falls per 100 woman years; $\alpha=0.05 ; 15 \%$ dropout rate). About eight months after recruitment began, however, we had to revise our target because of delays in this process in some centres. These delays were related mainly to the difficulties of simultaneously finding a nurse to perform the selection examinations, a room in the examination centre, and premises in the community for the exercise sessions. Accordingly, we could not open centres successively as we had planned initially. These logistic difficulties were compounded by the fact that the period of recruitment at each centre was limited to two to three months because a delay between recruitment and the first exercise class was likely to result in loss of motivation. On the other hand, because women had to live close to the intervention premises to be able to attend exercise classes every week, the geographical zone of recruitment could not be enlarged (without adding centres, which we were unable to do).

Given these time and geographical constraints and logistic problems, we decided to revise our initial main outcome. There is no consensus about the definition of an injurious fall outcome for intervention trials. For our initial sample calculation we had considered the most restrictive endpoint-that is, falls with a severe consequence such as a fracture or head trauma, which are relatively rare events. Falls with more moderate clinical consequences, however, which are more common, can also lead to medical care and have important psychological and functional consequences in older people. ${ }^{32}$ Thus, we decided to expand the main outcome to the rate of all injurious falls-that is, those resulting in both moderate and severe injuries. This enabled us to reduce the necessary sample size without modifying any other aspects of the trial (in particular, the inclusion criteria were not changed). The sample size was recalculated accordingly (change approved by the institutional review board of the study sponsor "DRCD-Assistance Publique-Hôpitaux de Paris" and the scientific expert jury for "Programme Hospitalier de Recherche Clinique PHRC National 2010" that extended funding for the study). We calculated that we needed 355 women in each group followed for two years to be able to show a 25\% reduction in all injurious falls (assumptions: 30 injurious falls per 100 woman years; $\alpha=0.05 ; \beta=0.15$; $15 \%$ dropout rate). ${ }^{33}$

\section{Statistical analysis}

Data were analysed according to the intention to treat principle. To examine the intervention's effectiveness, we started by graphically comparing the intensity of events (injurious falls) between the two groups by a mean cumulative function, $M C F(t)$, which shows the mean number of events per woman occurring up to time $t .^{34}$ The difference between the two mean cumulative functions corresponds to the average number of events per woman that the intervention would prevent within a given period. We used a log rank test of equality of the rate functions to test the equality of the two mean cumulative functions under the null hypothesis (assuming the weight functions were constant over time). ${ }^{35}$

The average event rates in the two groups over the two year study period were then modelled and compared with a "shared frailty" model, an extension of the Cox proportional hazard model that includes a frailty term (a statistical expression for random effect, unrelated to the clinical concept of frailty) to take dependency of events within an individual (correlation between different falls by the same person) into account. ${ }^{3637}$ All participants were included in the model until their last time point, which means that the model used all available data right up to the time of withdrawal or trial completion. For each fall outcome, we present the hazard ratios and 95\% confidence intervals for the intervention, adjusted for centre.

To assess the potential impact of attrition (incomplete outcome data) on the estimates of the effect of the 
intervention, we performed a sensitivity analysis using a worst case scenario based on the method proposed in the Cochrane review of interventions to prevent falls. ${ }^{4}$ We first calculated a rate ratio of falls (RaR1) by dividing the rate of injurious falls (injurious falls per person years) in the intervention group by the rate of injurious falls in the control group, using all available data. We estimated a second rate ratio of falls for all participants randomised ( $\mathrm{RaR} 2$ ) by using the conservative assumption that participants with missing data in the intervention group had the same rate of falls as observed in the control group (during their "lost" follow-up time) and vice versa. We then compared the two rate ratios. A ratio of these rate ratios (RaR2/RaR1) $>1.15$ or $<0.85$ indicates the possibility of clinically important bias.

Secondary outcomes were analysed with marginal (unconditional or population averaged) models, with a random effect for the study centre and an unstructured covariance structure for the residuals. All analyses were conducted with SAS 9.4 (SAS Institute).

\section{Results}

Trial participants and follow-up

Recruitment took place between December 2007 and May 2011, and follow-up ended in June 2013. Table 1 presents baseline demographic and health related characteristics of participants in the two groups. Figure 1 describes the flow of participants through the trial. Around $11 \%$ of the women invited to a free balance examination responded positively and were examined $(n=4221)$. Almost two thirds of the eligible women agreed to participate and were randomised to either the

\begin{tabular}{|c|c|c|}
\hline & Control $(n=354)$ & Intervention $(\mathrm{n}=352)$ \\
\hline Age (years) & $79.6(2.8)$ & $79.8(2.8)$ \\
\hline $\mathrm{BMI}\left(\mathrm{kg} / \mathrm{m}^{2}\right)$ & $27.0(4.6)$ & $27.1(4.6)$ \\
\hline No (\%) who fell at least once in past year & $159(45)$ & $137(39)$ \\
\hline No (\%) who used psychotropic drugs & $140(40)$ & $149(42)$ \\
\hline No (\%) who lived alone & $230(65)$ & $248(71)$ \\
\hline No (\%) who finished high school (baccalaureate) & $131(38)$ & $151(44)$ \\
\hline Visual acuity* & $6.9(2.2)$ & $6.9(2.1)$ \\
\hline Timed get up and go (s) & $12.4(3.1)$ & $12.4(2.7)$ \\
\hline Time to walk 6 m (s) & $7.5(1.7)$ & $7.4(1.7)$ \\
\hline No (\%) unable to do four tandem steps & $83(24)$ & $79(22)$ \\
\hline Time for the five chair stands (s) & $15.5(4.6)$ & $15.5(4.4)$ \\
\hline Time spent walking for casual activitiest (h/week) & $3.1(2.4)$ & $2.8(2.3)$ \\
\hline Median time (h/week) spent walking for exercise (IQR) & $0.75(0-3.3)$ & $0(0-3.4)$ \\
\hline $\begin{array}{l}\text { Median time (h/week) spent doing physical leisure } \\
\text { activities } \neq(I Q R)\end{array}$ & $1.5(0-3.5)$ & $1.0(0-3.4)$ \\
\hline Fear of falling score§ & $26.0(7.0)$ & $25.5(7.1)$ \\
\hline Physical functioning score & $57.5(20.7)$ & $59.4(21.6)$ \\
\hline General health score & $54.7(16.0)$ & $57.0(15.8)$ \\
\hline Psychological health score & $60.7(18.2)$ & $61.3(18.1)$ \\
\hline Vitality score & $46.6(16.1)$ & $48.44(16.3)$ \\
\hline \multicolumn{3}{|c|}{$\begin{array}{l}\text { IQR=interquartile range. } \\
\text { *Measured at distance of } 3 \text { m with Snellen letter test chart (decimal scale). } \\
\text { †Such as walking to general practitioner, pharmacy or store. } \\
\text { ¥Walking, swimming, dancing, gym classes, gardening, biking, and yoga. } \\
\text { §Based on the fall efficacy scale (FES-I). Score range16-64; higher score corresponds to more important concern } \\
\text { about falling. } \\
\text { १Based on SF-36 questionnaire. Score range 0-100; higher scores corresponds to less disability and better health.. }\end{array}$} \\
\hline
\end{tabular}

intervention ( $n=352)$ or the control group $(n=354)$. On average, participants in the intervention group were offered 94.9 (SD 11.6) exercise sessions over the entire intervention period; 58 (16.5\%) women never started the programme (fig 2). Among women who started the programme, the median number of sessions attended was 53 (interquartile range 16-71), and the median length of participation in the programme (from first to last attended session) was 79 weeks (32-97).

\section{Fall outcomes}

There were 397 injurious falls (in 189 women) in the control group and 305 (170 women) in the intervention group (table 2). Of all injurious falls, $14 \%$ were fractures and $8 \%$ were other serious injuries, while $78 \%$ had less severe consequences and were classified as moderate injurious falls (appendix 2). Over the two year intervention period, the injurious fall rate was 19\% lower in the intervention group than in the control group (hazard ratio $0.81,95 \%$ confidence interval 0.67 to $0.99 ; \mathrm{P}=0.04$ ). The rates of moderate and serious falls decreased by around the same order of magnitude (table 2). The estimated effect of the intervention for all falls was somewhat lower (0.88, 0.77 to $1.00 ; \mathrm{P}=0.05)$.

The mean cumulative function curves of injurious falls for the two groups began to separate between three and four months (fig 3). The difference in curves increased steadily until about nine months, then tended to plateau or increase more slowly over time (fig 4). By the end of the intervention, the average number of prevented injurious falls per participant was about 0.24 (95\% confidence interval 0.02 to 0.47 ). The mean cumulative function for all falls had progression similar to that of injurious falls (not shown).

The sensitivity analysis based on a worst case scenario led to a ratio of RaR1/RaR2 equal to 0.96 which, according to the Cochrane judgment rules, indicates limited potential for bias related to missing data on falls because of attrition.

\section{Other outcomes related to falls and health}

The intervention group fared significantly better than the control group in all balance and gait performance tests at one and two years (table 3 ). For most tests, results improved significantly in the intervention group, whereas there was no significant change in the control group. Fear of falling increased significantly in both groups but less so in the intervention group. The mean difference between groups in the FES-I score was significant at one year. No difference between the two groups was detected for physical activity indicators (table 4). All four health related quality of life scores tended to decrease in both groups (health status perceived as worsening over time) but less so in the intervention group (table 5). The mean difference between groups was significant for vitality and general health at one year and for physical function at one and two years.

\section{Adverse events}

Seven adverse events were reported in the intervention group. Four happened during group exercise sessions 


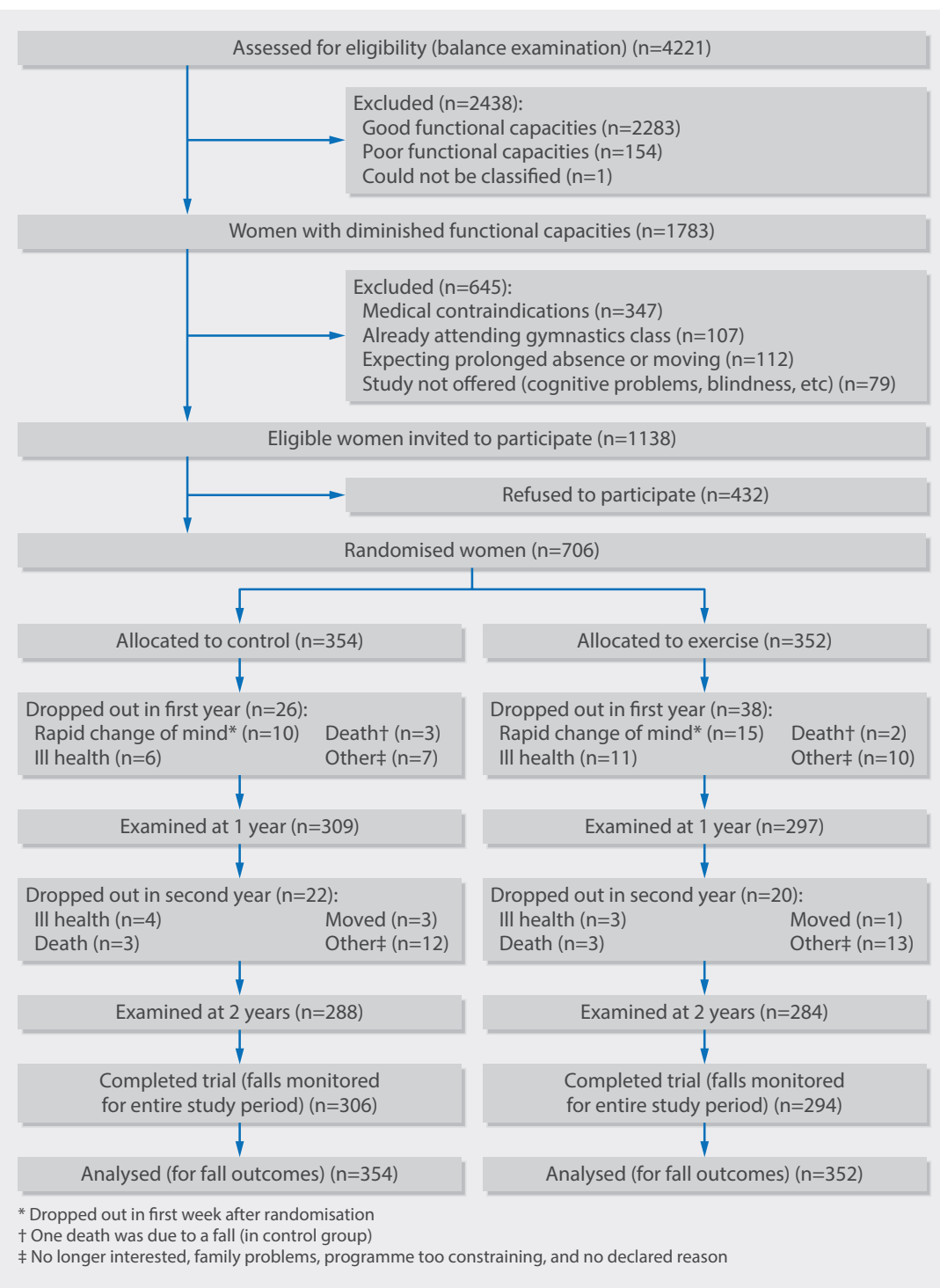

Fig 1 | Flow through trial of women aged 75-85 who took part in two year balance training programme for prevention of fall induced injuries (intervention) or no such programme (control)

\section{Discussion}

Fall outcomes

A two year programme of balance retraining significantly reduced the rate of injurious falls by $19 \%$ in women with an average age of 80 at risk of falling. The reduction in the most serious of those falls (such as those leading to a fracture) was of the same order of magnitude, though not significant, probably because of lack of power.

The programme's estimated effects on falls and injurious falls seem lower than the pooled average effects of exercise found in our meta-analysis. ${ }^{6}$ It is noteworthy that most of the interventions considered in this meta-analysis lasted less than a year (average 9.4 months), and few included very old people $(>75)$ at increased risk of falls and traumas. The lower effectiveness of the Ossébo programme could be related to its length and to the way its technical content evolved over time. The Ossébo programme was designed to ensure a progressive increase in the level and intensity of training while preserving achieved gains over time. The inflection of the difference between the mean cumulative function curves of the two comparison groups starting towards the end of the first year of intervention suggests that the second part of the programme could be re-adjusted to further improve balance and reduce injurious falls. There might be limits, however, in the extent to which balance and strength can be improved in older at risk subgroups. In any case, the maintenance of a positive difference between the mean cumulative function curves over time attests to the effectiveness of the programme in maintaining achieved progress.

Incomplete participation in the exercise programme might also have contributed to underestimation of the programme's effectiveness. In particular, a significant number of women in the intervention group never started the programme $(58 / 352,16.4 \%)$ or attended only a few classes and then stopped coming during the first month (38/352, 11\%). Most (81/96) nonetheless agreed to continue follow-up for falls. We had no systematic protocol to record reasons for withdrawal from the exercise programme for women who did not completely withdraw from the study (that is, with continued follow-up of falls). In the first two centres (in Amiens and Lille), one co-author (PDM) personally called women to investigate their reasons for not coming to sessions and low adherence to the programme. The most common reason was related to transportation problems in reaching the training site. This result confirms the field experience of SIEL Bleu instructors and other researchers who have set up fall prevention exercise trials (such as Freiberger and colleagues ${ }^{38}$ ) and is consistent with the barriers to participation reported by older people. ${ }^{39} 40$ The Ossébo trial is meant to have a pragmatic approach examining effectiveness rather than efficacy. A "per protocol" analysis, however, might also be informative as to the efficacy of the intervention. The definition of per protocol in the present case, though, is debatable. In particular, the groups to be compared in a per protocol analysis are not clear as women in the control group did not receive a placebo intervention (such as weekly

Fig 2 Details of participation of women aged 75-85 in two year balance training programme for prevention of fall induced injuries. IQR=interquartile range 
Table 2 | Consequences of falls and estimates of effect intervention (two year balance training programme for prevention of fall induced injuries) in women aged 75-85

\begin{tabular}{|c|c|c|c|}
\hline Consequences & $\begin{array}{l}\text { Control } \\
(n=354)\end{array}$ & $\begin{array}{l}\text { Exercise } \\
(\mathrm{n}=352)\end{array}$ & $\mathrm{HR}^{\star}(95 \% \mathrm{Cl})$ \\
\hline Total No of falls (ratet) & $640(0.92)$ & $533(0.79)$ & $0.88(0.77$ to 1.00$)$ \\
\hline No of participants who had at least one fall & 222 & 189 & - \\
\hline \multicolumn{4}{|l|}{ No of injurious falls (ratet): } \\
\hline Total & $397(0.56)$ & $305(0.45)$ & 0.81 (0.67 to 0.99$)$ \\
\hline Moderate & $310(0.44)$ & $237(0.35)$ & 0.81 (0.65 to 1.00$)$ \\
\hline Serious & $87(0.12)$ & $68(0.10)$ & $0.83(0.60$ to 1.16$)$ \\
\hline No of participants who had at least one injurious fall & 189 & 170 & - \\
\hline \multicolumn{4}{|c|}{$\begin{array}{l}\text { *Hazard ratio (adjusted for centre) computed with a "shared frailty" model. All women were included in analysis } \\
\text { until their last time point. Covariance for random effect: } 0.17 \text { ( } S E 0.04 ; \mathrm{P}<0.001 \text { ) for all falls; } 0.53 \text { (SE } 0.09 \text {; } \\
\mathrm{P}<0.001 \text { ) for injurious falls; } 0.57 \text { (SE } 0.10 ; \mathrm{P}<0.001 \text { ) for moderate injurious falls; } 0.35 \text { (SE } 0.25 ; \mathrm{P}=0.30 \text { ) for severe } \\
\text { injurious falls. } \\
\text { tRate per woman year=total number of events (fall related outcomes) divided by total number of woman years of } \\
\text { follow-up in each group. }\end{array}$} \\
\hline
\end{tabular}

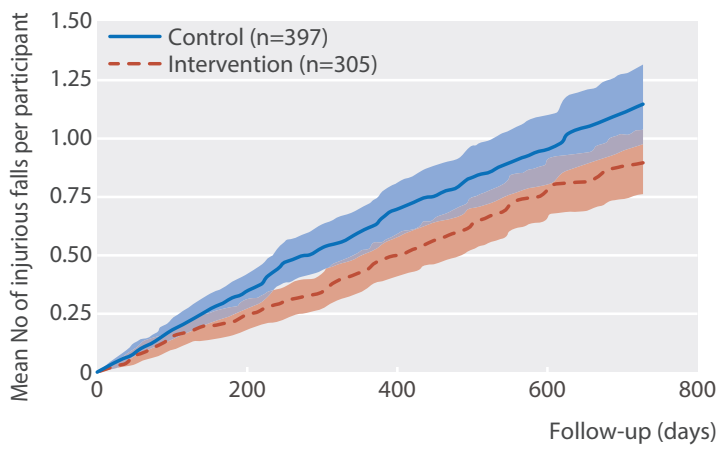

Fig 3 Mean cumulative function (MCF) for two comparison groups: mean number of injurious falls in women aged 75-85 in two year balance training programme for prevention of fall induced injuries (intervention) or no such programme (control)

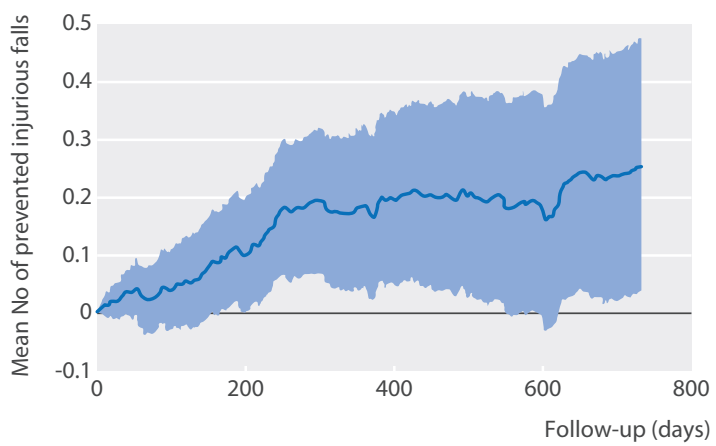

Fig 4 Difference in mean cumulative function (MCF) between two comparison groups: mean number of injurious falls prevented in women aged 75-85 in two year balance training programme for prevention of fall induced injuries

sessions of a low intensity exercise programme not designed to modify the risk of falling). Moreover, participation in the programme was rarely consistent, with most participants having variable bouts of absence throughout the intervention. We conducted a complementary and exploratory analysis, excluding from the intervention group women who never started the programme and adjusting for baseline fall risk factors. The resulting adjusted hazard ratio for injurious falls was 0.76 (95\% confidence interval 0.65 to 0.90 ).

Another possible explanation for our programme's lower effect compared with the pooled effect of exercise found in our recent meta-analysis could be related to the larger size of this trial and its multicentre nature (20 centres). A recent meta-epidemiological study found that single centre randomised controlled trials show larger treatment effects than multicentre trials, even after adjustment for sample size. ${ }^{41}$ One possible explanation for these findings is that the intervention implementation process might be less homogeneous in multicentre than in single centre studies. Selective outcome reporting in published studies (publication bias) is another possible explanation.

\section{Other outcomes related to falls and health}

We found that women in the intervention group significantly improved their balance and gait performances, which probably explains, at least in part, the beneficial effect of the Ossébo exercise programme. As in other studies though, ${ }^{42}$ improvements in test results seem relatively modest. Even small improvements in measured physical performances, however, could have important beneficial effects in older and already somewhat fragile people. In support of this hypothesis, we found that by the end of the intervention, women in the intervention group had a significantly better perception of their overall physical function (as assessed by the SF-36 questionnaire) than women in the control group, which suggests that improvements in physical performance have translated into improvements in global daily life functions. Moreover, while fear of falling increased significantly in both groups over the two year study period, this increase was less pronounced in the intervention group, which suggests that the programme is effective in limiting the age related decrease in older people's confidence in their ability to perform activities of daily living without falling. We found no significant differences in mean physical activity indicators between the two groups, which could be explained by the difficulty in making voluntary lifestyle changes in old age. Our activity indicators did not include participation in the Ossébo programme itself (which necessarily increased the overall activity level of the participants). The social contact inherent in attending the group classes and the motivation to get out to these classes every week could also have contributed to the beneficial effect of the programme.

\section{Strengths and limitations}

The study has several strengths. We used a well defined classification of injurious falls, selected before data collection. Incident falls were classified by an investigator blinded to group assignment, and many (73\%) of the serious injuries were validated by obtaining radiographs or hospital records. Our main outcome-injurious falls-is less likely to be biased because of under-reporting than the outcome of total falls. The trial had a pragmatic approach: the population selection criteria were simple and can easily be used by family physicians as well as health or physical activity practitioners providing care for older people; the intervention was implemented through a network of community based instructors with moderate levels of 


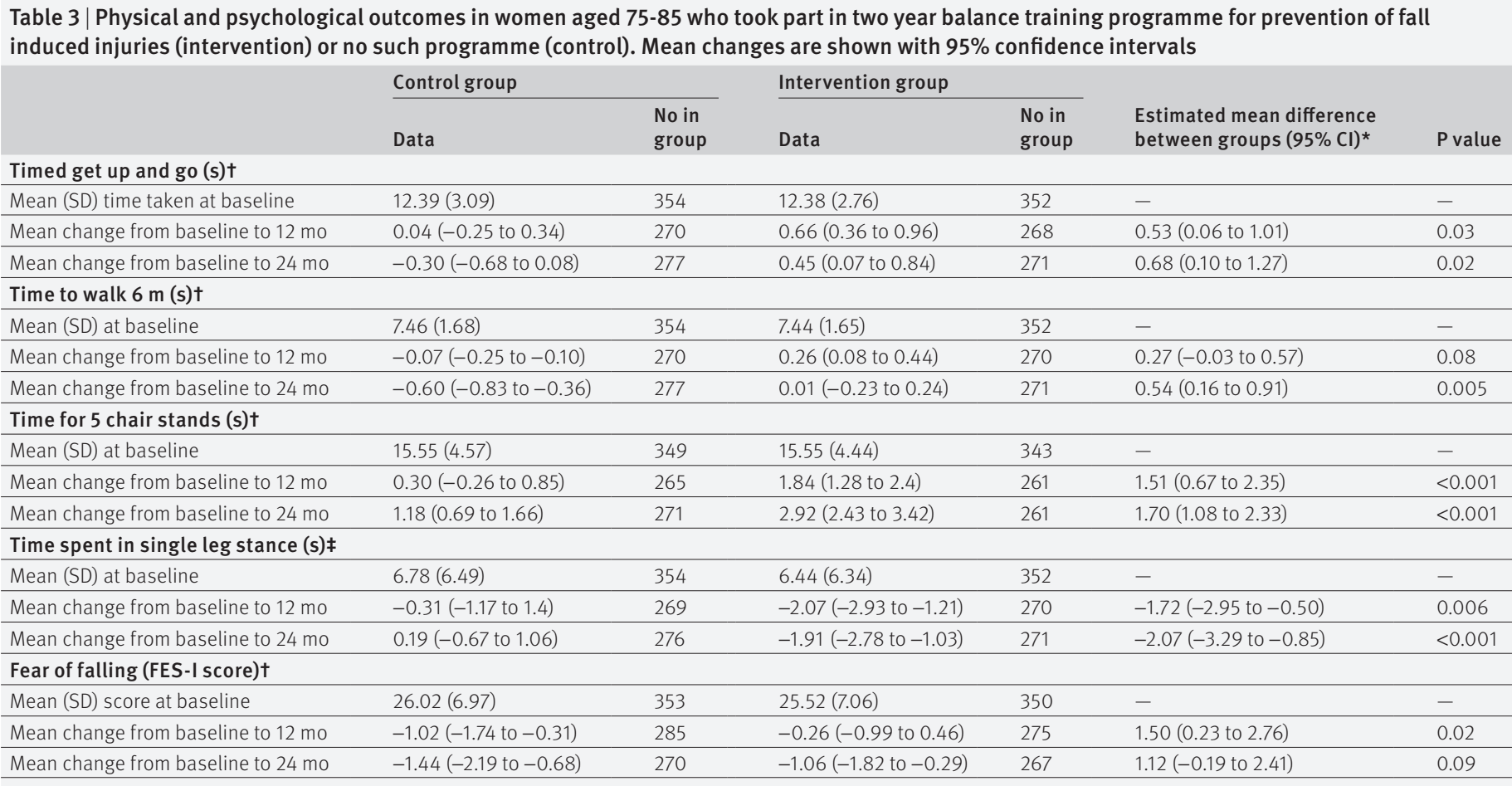

*Computed from unadjusted marginal model with random effect for centre.

†Positive mean difference between groups indicates better performance in intervention group.

‡Negative mean difference between groups indicates better performance in intervention group.

Table 4 | Physical activity outcomes in women aged 75-85 who took part in two year balance training programme for prevention of fall induced injuries (intervention) or no such programme (control). Mean changes are shown with $95 \%$ confidence intervals

\begin{tabular}{|c|c|c|c|c|c|c|}
\hline & \multicolumn{2}{|l|}{ Control group } & \multicolumn{2}{|l|}{ Intervention group } & \multirow[b]{2}{*}{$\begin{array}{l}\text { Estimated mean difference* } \\
\text { between groups }(95 \% \mathrm{Cl}) \dagger\end{array}$} & \multirow[b]{2}{*}{ Pvalue } \\
\hline & Data & $\begin{array}{l}\text { No in } \\
\text { group }\end{array}$ & Data & $\begin{array}{l}\text { No in } \\
\text { group }\end{array}$ & & \\
\hline \multicolumn{7}{|c|}{ Walking for casual activities (No/week) } \\
\hline Mean at baseline (SD) & $4.77(2.17)$ & 354 & $4.33(2.24)$ & 352 & - & - \\
\hline Mean change from baseline to $12 \mathrm{mo}$ & 0.33 (0.08 to 0.57$)$ & 309 & $0.23(-0.02$ to 0.48$)$ & 295 & $0.31(-0.05$ to 0.67$)$ & 0.09 \\
\hline Mean change from baseline to $24 \mathrm{mo}$ & $0.48(0.21$ to 0.75$)$ & 287 & $0.16(-0.10$ to 0.44$)$ & 281 & $0.10(-0.29$ to 0.48$)$ & 0.64 \\
\hline \multicolumn{7}{|l|}{ Walking for casual activities $¥$ (h/week) } \\
\hline Mean at baseline (SD) & $3.06(2.44)$ & 354 & $2.78(2.32)$ & 352 & - & - \\
\hline Mean change from baseline to $12 \mathrm{mo}$ & $-0.14(-0.42$ to 0.13$)$ & 309 & $-0.04(-0.33$ to 0.24$)$ & 295 & $0.39(-0.01$ to 0.79$)$ & 0.05 \\
\hline Mean change from baseline to $24 \mathrm{mo}$ & $-0.30(-0.64$ to 0.04$)$ & 287 & $-0.55(-0.9$ to -0.20$)$ & 281 & $0.04(-0.40$ to 0.48$)$ & 0.86 \\
\hline \multicolumn{7}{|l|}{ Walking for exercise (No/week) } \\
\hline Mean at baseline (SD) & $4.09(2.32)$ & 199 & $3.80(2.45)$ & 183 & - & - \\
\hline Mean change from baseline to $12 \mathrm{mo}$ & $-0.02(-0.42$ to 0.36$)$ & 181 & $0.00(-0.4$ to 0.4$)$ & 186 & $0.36(-0.11$ to 0.84$)$ & 0.13 \\
\hline Mean change from baseline to $24 \mathrm{mo}$ & $-0.03(-0.45$ to 0.38$)$ & 151 & $-0.24(-0.68$ to 0.18$)$ & 140 & $0.12(-0.40$ to 0.64$)$ & 0.65 \\
\hline \multicolumn{7}{|l|}{ Walking for exercise (h/week) } \\
\hline Mean at baseline (SD) & $2.11(2.82)$ & 354 & $1.89(2.89)$ & 352 & - & - \\
\hline Mean change from baseline to $12 \mathrm{mo}$ & $-0.19(-0.52$ to 0.13$)$ & 309 & $-0.37(-0.71$ to -0.04$)$ & 298 & $0.04(-0.45$ to 0.54$)$ & 0.86 \\
\hline Mean change from baseline to $24 \mathrm{mo}$ & $0.01(-0.32$ to -0.34$)$ & 286 & $-0.08(-0.42$ to 0.25$)$ & 279 & $0.13(-0.36$ to 0.63$)$ & 0.60 \\
\hline \multicolumn{7}{|c|}{ Leisure physical activities§ (No/week) } \\
\hline Mean at baseline (SD) & $1.02(1.21)$ & 354 & $0.99(1.32)$ & 352 & - & - \\
\hline Mean change from baseline to $12 \mathrm{mo}$ & $-0.29(-0.48$ to -0.11$)$ & 309 & $-0.30(-0.49$ to -0.11$)$ & 298 & $0.04(-0.25$ to 0.33$)$ & 0.79 \\
\hline Mean change from baseline to $24 \mathrm{mo}$ & $-0.23(-0.43$ to -0.03$)$ & 287 & $-0.02(-0.22$ to 0.17$)$ & 282 & 0.26 (0.00 to 0.52$)$ & 0.05 \\
\hline \multicolumn{7}{|l|}{ Leisure physical activities§ (h/week) } \\
\hline Mean at baseline (SD) & $2.43(2.86)$ & 354 & $2.23(3.06)$ & 352 & - & - \\
\hline Mean change from baseline to $12 \mathrm{mo}$ & $-0.38(-0.73$ to -0.03$)$ & 309 & $-0.50(-0.86$ to -0.15$)$ & 298 & $0.10(-0.43$ to 064$)$ & 0.70 \\
\hline Mean change from baseline to $24 \mathrm{mo}$ & $-0.11(-0.48$ to 0.25$)$ & 286 & $-0.07(-0.44$ to 0.29$)$ & 2801 & $0.27(-0.26$ to 0.80$)$ & 0.31 \\
\hline
\end{tabular}

*Negative mean difference between groups indicates better performance by intervention group.

tComputed from unadjusted marginal model with random effect for centre.

‡Such as walking to general practitioner, pharmacy, or store.

§Includes walking, swimming, dancing, gym classes, gardening, biking, and yoga. 


\begin{tabular}{|c|c|c|c|c|c|c|}
\hline \multirow[b]{2}{*}{ SF-36 score } & \multicolumn{2}{|l|}{ Control group } & \multicolumn{2}{|l|}{ Intervention group } & \multirow[b]{2}{*}{$\begin{array}{l}\text { Estimated mean difference* } \\
\text { between groups }(95 \% \mathrm{Cl}) \dagger\end{array}$} & \multirow[b]{2}{*}{$P$ value } \\
\hline & Data & $\begin{array}{l}\text { No in } \\
\text { group }\end{array}$ & Data & $\begin{array}{l}\text { No in } \\
\text { group }\end{array}$ & & \\
\hline \multicolumn{7}{|l|}{ Physical function } \\
\hline Mean at baseline (SD) & $57.47(20.72)$ & 353 & $59.40(21.06)$ & 351 & - & - \\
\hline Mean change from baseline to $12 \mathrm{mo}$ & 2.97 (1.02 to 4.92$)$ & 305 & $1.62(-0.36$ to 3.61$)$ & 294 & $-5.27(-9.06$ to -1.48$)$ & 0.01 \\
\hline Mean change from baseline to $24 \mathrm{mo}$ & 5.68 (3.81 to 7.56$)$ & 287 & 2.97 (1.06 to 4.87) & 281 & $-3.90(-7.51$ to -0.30$)$ & 0.03 \\
\hline \multicolumn{7}{|l|}{ Mental health } \\
\hline Mean at baseline (SD) & $60.70(18.24)$ & 354 & $61.33(18.1)$ & 350 & - & - \\
\hline Mean change from baseline to $12 \mathrm{mo}$ & $0.78(-0.81$ to 2.38$)$ & 304 & $-1.19(-2.83$ to 0.44$)$ & 291 & $-2.55(-5.33$ to 0.25$)$ & 0.07 \\
\hline Mean change from baseline to $24 \mathrm{mo}$ & $0.13(-1.44$ to 1.72$)$ & 284 & $-0.87(-2.48$ to 0.73$)$ & 281 & $-1.58(-4.26$ to 1.11$)$ & 0.25 \\
\hline \multicolumn{7}{|l|}{ General health } \\
\hline Mean at baseline (SD) & $54.72(16.05)$ & 353 & $57.05(15.80)$ & 349 & - & - \\
\hline Mean change from baseline to $12 \mathrm{mo}$ & $-0.25(-1.71$ to 1.21$)$ & 301 & $-0.69(-2.19$ to 0.80$)$ & 287 & $-2.33(-4.86$ to 0.20$)$ & 0.04 \\
\hline Mean change from baseline to $24 \mathrm{mo}$ & $1.16(-0.37$ to 2.70$)$ & 282 & $1.40(-0.15$ to 2.97$)$ & 280 & $-1.64(-4.21$ to 0.93$)$ & 0.21 \\
\hline \multicolumn{7}{|l|}{ Vitality } \\
\hline Mean at baseline (SD) & $46.62(16.15)$ & 350 & $48.44(16.26)$ & 350 & - & - \\
\hline Mean change from baseline to $12 \mathrm{mo}$ & $1.17(-0.35$ to 2.70$)$ & 303 & $-0.36(-1.92$ to 1.19$)$ & 290 & $-3.17(-5.71$ to -0.62$)$ & 0.01 \\
\hline Mean change from baseline to $24 \mathrm{mo}$ & 1.65 (0.04 to 3.26$)$ & 285 & $0.71(-0.91$ to 2.34$)$ & 281 & $-2.57(-5.21$ to 0.08$)$ & 0.06 \\
\hline
\end{tabular}

training and expertise (a post-secondary course of at least three years in physical and sports training) and was delivered in settings representative of future target settings, which should facilitate generalisation of the programme.

There were, however, also several limitations that must be acknowledged. Data on falls were missing (incomplete) for 105 (14.9\%) participants who either died (six in the control group, five in the interventions group) or withdrew from the study ( 42 and 53, respectively) at various points. In both groups, the number of dropouts was larger the first year than in the second year ( $9 \%$ overall at one year, which is equivalent to the median 12 month attrition rate reported in other fall prevention exercise trials). ${ }^{43}$ This was especially true in the intervention group (38 $v 26$ during the first year), with quite a few women rapidly changing their minds and withdrawing from the study within a few days of their inclusion (10 and 15, respectively). This is not really surprising as women had to decide if they wanted to participate at the end of the baseline examination, but several of them might have realised only later on the extent of commitment associated with participation in the trial or were afraid or embarrassed to tell the nurse examiner that they did not want to participate. For women in the intervention group, the constraints attached to participation were even greater, especially as they could not chose the day or time of the weekly exercise classes. As noted in the Cochrane review, ${ }^{4}$ the reasons usually offered for dropping out of fall prevention trials are diverse (no longer interested, no benefit for control participants, too constraining for intervention participants, family problems, too busy, too tired, illness unrelated to falls, etc), and it is difficult to know how these combined (and self reported) reasons actually affected the true rate of falls in each comparison group. Sensitivity analysis with a worst case scenario suggests that the risk of bias from attrition is probably low.
Another limitation of this study is that it is underpowered to definitively show an effect on the most severe injurious falls. This is partly because of the logistic complexity of setting up large multicentre studies, ${ }^{4445}$ but also perhaps because of the age of the target population and the well documented difficulty of recruiting older people in randomised controlled trials. ${ }^{46}$ This difficulty is compounded by the fact that older adults tend to deny or underestimate their risk of falling and show little interest in fall prevention programmes, 4047 which probably helps to explain the low response rate to our initial invitation to a balance examination. Similar participation rates have been reported in other randomised controlled trials of fall prevention programmes. ${ }^{2628}$

The Ossébo exercise programme targeted women aged 75-85 who were at moderate risk of falls and injuries (neither too fit nor too frail). Although balance training programmes can be regarded as appropriate for most women aged 75-85 living in the community, making groups more homogeneous allows better adaptation of the programme to the physical capacities of women in the group and to maintain motivation over time of all women within the group. Furthermore, many older people think that individual variations within groups are a disadvantage. ${ }^{48}$

Not unexpectedly, the trial participants were more likely to have had a fall in the previous 12 months than the general population of women of same age $(40 \% \mathrm{v}$ $33 \%),{ }^{49}$ which indicates that our sample is more representative of the intended target population than of the overall sex and age matched population. Among the women examined and eligible for the trial, risk of falls (based on history of falls, fear of falling, physical performances, and perceived physical health) did not differ significantly between those who accepted and those who refused to participate, which indicates that no additional self selection (based on fall risk) occurred 
above and beyond our intended selection process. Participants' baseline characteristics varied substantially in perceived health status, physical capacities, and physical activity. Hence, the Ossébo intervention should be relevant to many at risk older women in the population at large. On the other hand, comparison with census data indicates that the trial's participants had a higher education level than women aged 75-85 in the general population ( $40 \% \mathrm{v} 12 \%$ had finished high school), which is to be expected in studies of volunteers, and were more likely to live alone ( $68 \%$ v $48 \%) .50$ These points suggest that future studies should develop and test different strategies to reach out to a larger number of older women who could benefit from the Ossébo programme, in particular in more disadvantaged communities (for example, through involvement of general practitioners and use of participatory methods) and to improve arguments used to motivate older people to participate (for example, by taking better account of familial constraints or emphasising the possibility of meeting other people in a convivial atmosphere). Characteristics of the programme also influence the decision of older people to participate ${ }^{4051}$ and point to several other possible levers for increasing participation (such as provision of free transportation to the exercise premises, as suggested by some of the non-starters and early dropouts in our study).

In conclusion, our study shows that it is feasible to put into place a large scale, long term fall prevention exercise programme that is effective in reducing injurious falls among older at risk women. The programme improved balance and gait capacities and also quality of life related to physical function for this group of older women. Future studies should assess different strategies aimed at increasing the level of outreach to the target population and at improving participation.

We thank the directors of the clinical investigation centres who helped in the conduct this study (J Arvieu (Boulogne-Billancourt), P L Bernard (Montpellier), G Berrut (Nantes), F Blanchard (Reims), M Bonnefoy (Lyon), E Bouvard (Paris 75020), B Cassou (Paris 75016), S DebrayMeignan (Paris 75005), C Debure (Issy-les-Moulineaux), P Dejardin (Paris 75017), M Dramé (Reims), P Fardellone (Amiens), R Gonthier (Saint Etienne), C Jeandel (Montpellier), I Lebrun (Reims), S Liabeuf (Amiens), P Livet (Lyon), E Maggia (Nîmes), C Marcelli (Caen), C Martin-Hunyadi (Strasbourg), S Moussay (Caen), V Poquet (Annecy), F Puisieux (Lille), J P Taillandier (Villejuif), J Weber (Rouen), and Y Wolmark (Paris 75018)). We also thank the clinical research assistants who helped in the implementation and coordination of the study ( $S$ Emprin, G Gonzalez, M Borsato) and the clinical examiners (J Pasek, H Fleury, S Gaudioz, K Castro-Lionard, A Caravati, A Ponoran, T Martin, L D’Agui, I Pollet, C Royes, M L Lepilliez). We are indebted to the SIEL Bleu group, in particular to J M Ricard and J D Muller (directors), D Lutz (Ossébo exercise programme conception and monitoring), C Hénon, C Hiegel, and A Seroux (coordinators of the Ossébo programme implementation), and all the SIEL Bleu instructors who implemented the intervention in the study centres. We specially thank P MartelSamb and C Aubrière, who developed the software programme for the computerised randomisation and data collection processes (AP-HP, Hôpital Ambroise Paré, Unité de Recherche Clinique), and A Ouslimani and E Soler (DRCD, AP-HP), who helped us with the study financial dossier and administrative authorisation demands (ethics committee, CNIL). We also thank J Pénichoux and B Heude (CESP, INSERM U1018, Villejuif) for their advice regarding the statistical analysis. We are grateful to I Boutron (Cochrane centre, France; Centre de Recherche Epidémiologie et Statistique Sorbonne-Paris-Cité (CRESS), UMR1153, Paris, Inserm-Université Paris Descartes) for her helpful comments and advice regarding the final manuscript and Jo Ann Cahn for her help in preparing the manuscript. Finally, we thank all the women who agreed to participate in the study.

Contributors: PDM and BC conceived and designed the trial, and supervised the conduct of the trial and the acquisition of data. PA advised on the randomisation procedure and other statistical aspects. FEK analysed the data with the assistance of PDM. AL advised on and reviewed statistical analysis. FEK and PDM drafted the final manuscript. All co-authors were involved in the interpretation of the results and contributed to reviewing the manuscript. PDM is guarantor.

Funding: This study was sponsored by "Assistance Publique-Hôpitaux de Paris" (AP-HP). The project funded by grants from the French Ministry of Health (Programme Hospitalier de Recherche Clinique PHRC National, 2006: AOM06076, 2010: AOM10144), the French National Research Agency (ANR) (BLAN06-3_135165), the National Institute of Health Prevention and Education (INPES) (project No 047/06-DAS), and the Council of the Ile-de-France region (Partnership of Citizens and Institutions for Research and Innovation, PICRI, No 09003420). The funders had no role in study design, data collection, and analysis, the decision to publish or preparation of the manuscript. PD-M received a "Contrat d'Interface vers l'hôpital" (interface Inserm-Hospitals) from the hospital group "Ste Périne, ChardonLalache, Rossini” (Assistance Publique-Hôpitaux de Paris (AP-HP)) in June 2008.

Competing interests: All authors have completed the Unified Competing Interest form at http://www.icmje.org/coi_disclosure.pdf (available on request from the corresponding author) and declare: no support from any organisation for the submitted work; no financial relationships with any organisations that might have an interest in the submitted work in the previous three years; no other relationships or activities that could appear to have influenced the submitted work.

Ethical approval: The study protocol was approved by the Ile-de-France IV Committee for the Protection of Persons (CPP) (ref 2007/29). It is registered with the CNIL (French Data Protection Authority) under No 907198. Each woman received written information about the study (under French law, written consent is not required for this intervention, which is considered as "usual care").

Transparency: The lead author (the manuscript's guarantor) affirms that the manuscript is an honest, accurate, and transparent account of the study being reported; that no important aspects of the study have been omitted; and that any discrepancies from the study as planned (and, if relevant, registered) have been explained.

Data sharing: No additional data available.

This is an Open Access article distributed in accordance with the Creative Commons Attribution Non Commercial (CC BY-NC 4.0) license, which permits others to distribute, remix, adapt, build upon this work non-commercially, and license their derivative works on different terms, provided the original work is properly cited and the use is non-commercial. See: http://creativecommons.org/licenses/ by-nc/4.0/.

1 Rubenstein LZ. Falls in older people: epidemiology, risk factors and strategies for prevention. Age Ageing 2006;35(suppl 2):37-41.

2 Kannus P, Sievänen $H$, Palvanen M, et al. Prevention of falls and consequent injuries in elderly people. Lancet 2005;366:1885-93.

3 Gill TM, Murphy TE, Gahbauer EA, et al. Association of injurious falls with disability outcomes and nursing home admissions in community-living older persons. Am J Epidemiol 2013;178:418-25. Gillespie LD, Robertson MC, Gillespie W], et al. Interventions for preventing falls in older people living in the community. Cochrane Database Syst Rev Online 2012;9:CD007146.

5 Sherrington C, Whitney JC, Lord SR, et al. Effective exercise for the prevention of falls: a systematic review and meta-analysis. J Am Geriatr Soc 2008;56:2234-43

6 El-Khoury F, Cassou B, Charles M-A, et al. The effect of fall prevention exercise programmes on fall induced injuries in community dwelling older adults: systematic review and meta-analysis of randomised controlled trials. BMJ 2013;347:f6234.

7 Lamb SE, Jørstad $\mathrm{S}$ Stein EC, Hauer K, et al. Development of a common outcome data set for fall injury prevention trials: the Prevention of Falls Network Europe Consensus. J Am Geriatr Soc 2005;53:1618-22.

8 Robertson MC, Campbell AJ, Gardner MM, et al. Preventing injuries in older people by preventing falls: a meta-analysis of individual-level data. J Am Geriatr Soc 2002;50:905-11.

9 Close JCT. How can you prevent falls and subsequent fractures? Best Pract Res Clin Rheumatol 2013;27:821-34.

10 Rose DJ, Hernandez D. The role of exercise in fall prevention for older adults. Clin Geriatr Med 2010;26:607-31.

11 Dargent-Molina P, Khoury FE, Cassou B. The “Ossébo" intervention for the prevention of injurious falls in elderly women: background and design. Glob Health Promot 2013;20:88-93. 
12 Dargent-Molina P, Cassou B. Prévention des chutes et des fractures chez les femmes âgées : l'essai randomisé Ossébo. Gérontologie Société 2008:125:65-78.

13 Treweek S, Zwarenstein M. Making trials matter: pragmatic and explanatory trials and the problem of applicability. Trials 2009;10:37.

14 Zwarenstein M, Treweek S, Gagnier JJ, et al. Improving the reporting of pragmatic trials: an extension of the CONSORT statement. BMJ 2008;337:a2390.

15 Dargent-Molina P, Favier F, Grandjean H, et al. Fall-related factors and risk of hip fracture: the EPIDOS prospective study. Lancet 1996;348:145-9.

16 Lee SH, Dargent-Molina P, Bréart G. Risk factors for fractures of the proximal humerus: results from the EPIDOS prospective study. I Bone Miner Res 2002;17:817-25.

17 Leplège A, Ecosse E, Verdier A, et al. The French SF-36 health survey: translation, cultural adaptation and preliminary psychometric evaluation. J Clin Epidemiol 1998;51:1013-23.

18 Cummings SR, Nevitt MC, Browner WS, et al. Risk factors for hip fracture in white women. Study of Osteoporotic Fractures Research Group. N Engl I Med 1995;332:767-73.

19 Guralnik JM, Simonsick EM, Ferrucci L, et al. A short physical performance battery assessing lower extremity function: association with self-reported disability and prediction of mortality and nursing home admission. J Gerontol 1994;49:M85-94.

20 Podsiadlo D, Richardson S. The timed 'Up \& Go': a test of basic functional mobility for frail elderly persons. J Am Geriatr Soc 1991;39:142-8.

21 Yardley L. Development and initial validation of the Falls Efficacy Scale-International (FES-I). Age Ageing 2005;34:614-9.

22 Lord SR, Ward JA, Williams P, et al. The effect of a 12-month exercise trial on balance, strength, and falls in older women: a randomized controlled trial. J Am Geriatr Soc 1995;43:1198-206.

23 Delbaere K, Hauer K, Lord SR. Evaluation of the incidental and planned activity questionnaire (IPEQ) for older people. BrJ Sports Med 2010;44:1029-34.

24 Dargent-Molina P, Poitiers F, Bréart G. In elderly women weight is the best predictor of a very low bone mineral density: evidence from the EPIDOS study. Osteoporos Int 2000;11:881-8.

25 Tinetti ME, Baker DI, McAvay G, et al. A multifactorial intervention to reduce the risk of falling among elderly people living in the community. N Engl J Med 1994:331:821-7.

26 Campbell AJ, Robertson MC, Gardner MM, et al. Randomised controlled trial of a general practice programme of home based exercise to prevent falls in elderly women. BM/1997:315:1065-9.

27 Skelton D, Dinan S, Campbell M, et al. Tailored group exercise (Falls Management Exercise - FaME) reduces falls in community-dwelling older frequent fallers (an RCT). Age Ageing 2005;34:636-9.

28 Day L. Randomised factorial trial of falls prevention among older people living in their own homes. BMJ 2002;325:128.

29 Lord SR, Castell S, Corcoran J, et al. The effect of group exercise on physical functioning and falls in frail older people living in retirement villages: a randomized, controlled trial. J Am Geriatr Soc 2003;51:1685-92.

30 Jones CJ, Rose DJ. Physical activity instruction of older adults. Human Kinetics, 2005.

31 Buchner DM, Hornbrook MC, Kutner NG, et al. Development of the common data base for the FICSIT trials. J Am Geriatr SoC 1993:41:297-308.

32 Tinetti ME, Williams CS. Falls, injuries due to falls, and the risk of admission to a nursing home. N Engl J Med 1997;337:1279-84
33 Schlesselman JJ. Sample size requirements in cohort and case-control studies of disease. Am J Epidemiol 1974;99:381-4.

34 Donaldson MG, Sobolev B, Kuramoto L, et al. Utility of the mean cumulative function in the analysis of fall events. J Gerontol A Biol Sci Med Sci 2007;62:415-9.

35 Gutierrez RG. Good as new or bad as old? Analyzing recurring failures with the RELIABILITY procedure. SAS, 2013.

36 Box-Steffensmeier JM, De Boef S. Repeated events survival models the conditional frailty model. Stat Med 2006;25:3518-33.

37 Duchateau L, Janssen P. The frailty model. Springer Scienceand Business Media, 2007.

38 Freiberger E, Blank W, Salb J, et al. Effects of a complex intervention on fall risk in the general practitioner setting: a cluster randomized controlled trial. Clin Interv Aging 2013;8:1079-88.

39 Dickinson A, Machen I, Horton K, et al. Fall prevention in the community: what older people say they need. Br J Community Nurs 2011;16:174-80

40 Bunn F, Dickinson A, Barnett-Page E, et al. A systematic review of older people's perceptions of facilitators and barriers to participation in falls-prevention interventions. Ageing Soc 2008;28:449-72.

41 Dechartres A, Boutron I, Trinquart L, et al. Single-center trials show larger treatment effects than multicenter trials: evidence from a meta-epidemiologic study. Ann Intern Med 2011;155:39-51.

42 Howe TE, Rochester L, Jackson A, et al. Exercise for improving balance in older people. Cochrane Database Syst Rev 2011;11: CD004963.

43 Nyman SR, Victor CR. Older people's participation in and engagement with falls prevention interventions in community settings: an augment to the cochrane systematic review. Age Ageing 2012;41:16-23.

44 McDonald AM, Knight RC, Campbell MK, et al. What influences recruitment to randomised controlled trials? A review of trials funded by two UK funding agencies. Trials 2006; 7:9.

45 Stevens Z, Carpenter H, Gawler S, et al. Lessons learnt during a complex multicentre cluster randomised controlled trial: the ProAct65+ trial. Trials 2013;14:192.

46 Auster J, Janda M. Recruiting older adults to health research studies: a systematic review. Australas / Ageing 2009;28:149-51.

47 Yardley L, Donovan-Hall M, Francis K, et al. Older people's views of advice about falls prevention: a qualitative study. Health Educ Res 2006;21:508-17.

48 De Groot GCL, Fagerström L. Older adults' motivating factors and barriers to exercise to prevent falls. Scand J Occup Ther 2011;18:153-60.

49 Léon C, Beck F. Les comportements de santé des 55-85 ans analyses du Baromètre santé 2010. Inpes éd, 2014.

50 INSEE. Insee references-les personnes agees. Insee, 2005. www. insee.fr/fr/ppp/sommaire/PERSAG05.PDF.

51 Franco MR, Howard K, Sherrington C, et al. Eliciting older people's preferences for exercise programs: a best-worst scaling choice experiment. J Physiother 2015;61:34-41.

(C) BMJ Publishing Group Ltd 2015 Appendix 1: Details of the Ossébo fall and injury
prevention exercise programme

Appendix 2: Description of consequences associated with moderate and serious injurious falls 